\title{
The Package Server Location Problem
}

\author{
Arnaud Malapert ${ }^{1}$, Jean-Charles Régin ${ }^{1}$ and Jean Parpaillon ${ }^{2}$ \\ ${ }^{1}$ Laboratoire d'Informatique, Signaux et Systèmes de Sophia-Antipolis (I3S) \\ CNRS UMR7271, Université Nice Sophia Antipolis, Sophia Antipolis, France \\ ${ }^{2}$ Mandriva, 32 rue Trevise, 75009 Paris, France \\ firstname.lastname@unice.fr, jparpaillon@mandriva.com
}

Keywords: Multi-objective Optimization, Mixed Integer Linear Programming, Network Location Model.

\begin{abstract}
In this paper, we introduce a new multi-objective optimization problem derived from a real-world application: the package server location problem. A number of package servers are to be located at nodes of a network. Demand for these package servers is located at each node, and a subset of nodes are to be chosen to locate one or more package servers. Each client is statically associated to a package server. The objective is to minimize the number of package servers while maximizing the efficiency and the reliability of the broadcast of packages to clients. These objectives are contradictory: the broadcast becomes more efficient as the number of servers increases. This problem is analyzed as a multi-objective optimization problem and a mathematical formulation is proposed. In addition, the criteria combination can be specified via a small dedicated language. Results for exact multi-objective solution approaches based on mixed integer linear programming are reported.
\end{abstract}

\section{INTRODUCTION}

Allocating services to servers of an existing computer network is a complex and difficult task for the administrators. Indeed, services are interrelated on nodes of the network under several constraints (node capacities, incompatibilities between services ...). Moreover, the administrators consider simultaneously multiple and contradictory objectives or preferences (cost, performance, reliability, energy consumption). Thus, a decision tool which can provide solutions meeting these goals is valuable. Mandriva ${ }^{1}$, a French software company, must accomplish such a task in the deployment of one of their product. This company is well-known for its public open source distribution of operating system: Mandriva Linux. It also offers product solutions for companies to manage their computer network infrastructure. Pulse2 is an open-source tool that simplifies application deployment, inventory, and maintenance of computer networks. It helps organizations ranging from a few computers to more than 100000 heterogeneous computers spread over more than 1000 facilities to inventory, maintain, update and take full control on their facilities. On one hand, Pulse 2 helps manage networks of french governmental agencies or real estate offices. On the other hand, it also helps manage net-

\footnotetext{
${ }^{1}$ http://www.mandriva.com/
}

work mainly composed of gaming or ATM terminals. The user can supervise large-scale facilities through a single web interface console, create and deploy hard disk images of its computers, perform software and hardware inventory, and deploy new softwares and updates. Pulse 2 is installed on the top of an existing infrastructure and provides a cross-platform software distribution service even for remote locations with a limited bandwidth. The architecture of Pulse 2 is distributed: Pulse 2 is composed of agents communicating with each other by various protocols (http, ssh, $\mathrm{xml}-\mathrm{rpc}$, etc). An agent is a piece of software that is replicated and distributed over hosts of the network, and is usually continuously connected to a central job scheduler. Distributed agents improve the robustness and the scalability of Pulse2, but have two major drawbacks: finding a feasible deployment of agents on hosts is difficult; upgrading them can cause severe production downtime. Features of Pulse 2 are implemented through client-server models where computers and agents respectively play the role of clients and servers. In other words, Pulse 2 is a distributed application that allows the management by a central authority of application deployment and maintenance in a heterogeneous network.

An essential functionality of Pulse 2 is the management of software installations and updates, which can lead to network congestion and failures. A package server, abbreviated as pserver, maintains the list 
of software packages and serves them to its clients (computers). Each client is statically associated to a unique pserver by the central authority. Pulse2 allows to manage groups of clients thanks to its compatibility with other resource-manager such as GLPI ${ }^{2}$. For instance, research and administration computers can have different software suites or security requirements.

The deployment of pservers involves strategic decisions that are fundamental since they impact the efficiency and reliability of the broadcast of packages from pservers to clients when large software packages are installed or updated (such as an office suite) on hundreds or thousands of clients. An efficient broadcast reduces the completion time for serving all clients while avoiding network congestion. A reliable broadcast reduces the impact of link failures. Note that the flexibility of the design is important in view of uncertain scope of future additions to the network.

The main contribution of the paper is twofold. We describe a new multi-objective optimization problem derived from a real-world application: the package server location problem (PSLP). Then, we propose a flexible and efficient decision support tool based on operational research methods for multi-criteria optimization. This paper is organized as follows. In Section 2, we describe the package server location problem and discuss an example. In Section 3, we discuss related works in the field of mixed integer linear programming, cloud management, network design and facility location, especially server location in computer networks. In Section 4, we present some concepts and discuss related works in the field of multi-objective optimization. In Section 5, we introduce a mathematical formulation and discuss in Section 6 our method to handle multi-objective optimization within an integer programming framework. Finally, in Section 7, we show the interest of our approach on a realistic use case and evaluate systematically the impact of the criteria combination on the solving process.

\section{THE PACKAGE SERVER LOCATION PROBLEM}

\section{Description, Notations, and Definitions}

Tree Network. According to the Mandriva Team, tree network is the most frequent structure adopted by its customers. Let $N=[1, n]$ be the indices of the facilities organized as an oriented tree network

\footnotetext{
${ }^{2} \mathrm{http} / / / w w w . g l p i-p r o j e c t . o r g /$
}

$\mathcal{T}=(N, E)$. The pservers can be located at nodes (facilities) and the root node is called the central facility. Each network link $(i, j) \in E$ between a pair of facilities $i \in N$ and $j \in N$ is characterized by a real bandwidth $B_{i j}$ and a boolean reliability $r_{i j}$. The link bandwidth and reliability respectively allow to model network congestion and the probability of failure.

Client-server Model. Each client must be associated to a single pserver. Clients are located at facilities and partitioned into $g$ groups, i.e. each client belongs to a unique group. Let $d_{i}^{s}$ be the demand at the facility $i \in N$ for the group $s(s=1, \ldots, g)$. We assume that groups are treated independently by the central authority, i.e. packages are never installed or updated simultaneously for two groups.

For each type $k \in K(=[1, m])$ of pserver, the central authority determines its maximal number of clients (its capacity) $C_{k}$ in order to provide the same service quality. Let $M_{i k}$ be the maximal number of pservers of type $k \in K$ which can be located at facility $i$. The central authority determines $M_{i k}$ by monitoring the network.

Package Broadcast. A package is broadcasted in two steps as follows. First, the root pserver located at the central facility receives the original package and broadcasts it to other pservers. As soon as they have received the full copy of the original package, pservers serve their clients. The broadcast is oriented, i.e. a pserver can only serve clients located at the same facility or in its descendants. Let define a special group $(s=0)$ for which the demand $d_{i}^{0}$ is equal to the number of pservers located at the facility $i$. Let $S=[0, g]$ denote the stages where the initial stage $(s=0)$ represents the broadcast from the root pserver to other pservers and next stages represent the broadcast from pservers to groups of clients.

Broadcast Model. We consider only the steady state of the broadcast of the traffic rather than the transient state, i.e. we do not take into account transmission times and path latencies (the sum of all transmission times as well as the queuing inside the facilities). Indeed, the duration of the transient state is dominated by the maximal latency which is usually lower than the total duration of the broadcast. Furthermore, path latencies are hard to measure and more useful for operational decisions which affect the day-to-day implementation of these strategic decisions.

We assume that the steady state of the broadcast of the traffic follows a model such that a) transfers are synchronous, b) a bandwidth is allocated to each transfer path in the network, c) the path bandwidth is 
consumed simultaneously along its links during the transfer, d) the path bandwidth is shared equally by clients. Unlike distance, link bandwidths do not sum up in the path bandwidth. The path bandwidth is limited by the minimum of link bandwidths, as this is the fastest any traffic can make through the path. The link bandwidth is shared by the traffic under consideration. The total bandwidth allocated to paths sharing a link must not exceed the link bandwidth.

Figure 1 illustrates the broadcast model where facilities are represented as nodes, links as arcs between nodes, and paths as arrows besides the tree. In this example, the traffic passing through the dashed link is composed of the paths from its origin, or its ancestors, to its destination, or its descendants. The sum of path bandwidths must not exceed the bandwidth of the dashed link.

Similarly, the path reliability is limited by the less reliable link. If the path from the central facility to another facility is reliable, then the facility is said to be reliable. Note that we assume that local connections (the pserver and the client are located at the same facility) are reliable and more efficient than connections between facilities.

Objectives. In summary, solving the problem boils down to a) select the number of pservers, b) select the type of pservers, c) select the location of pservers, d) associate pservers to clients subject to various capacity and broadcast constraints. In general, we have several measures for the quality of the solutions. First, we aim to minimize the number of pservers or a cost function associated to their deployment. Then, we also aim to maximize the reliability of the transfer, i.e. maximizing the number of pservers located at reliable facilities and the number of reliable connec-

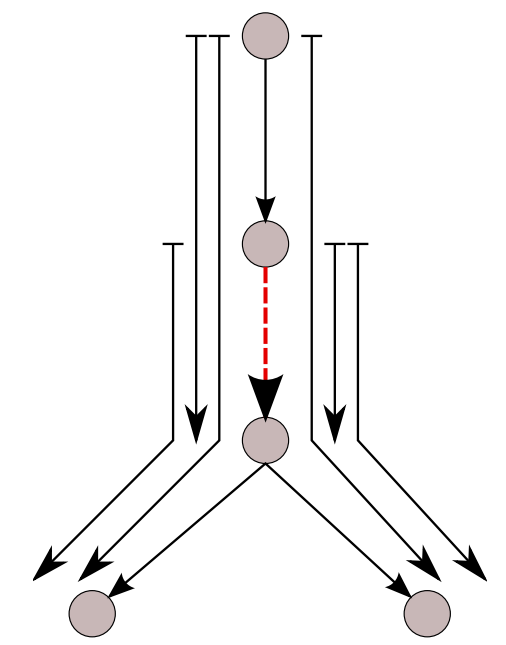

Figure 1: Broadcast model. tions between pservers and clients. Besides, we aim to maximize the efficiency of the broadcast of packages from pservers to clients by minimizing the total distance or maximizing the total allocated bandwidth. These objectives are contradictory: the broadcast becomes more efficient as the number of pservers increases. That is to say that PSLP, in its generality, is a multi-objective optimization problem. The ability to express the kind of trade-off we want depends on the solving method.

\section{Illustrative Example}

Figure 2 gives a complete example composed of a small instance and several solutions. The instance contains 6 facilities, 3 levels, 1 type of pservers, and 1 group of 2500 clients. We can install as many pservers as needed in facilities and each pserver provides 500 connections. The tree network is represented in Figure 2(a) where each facility $i$ is drawn as a record in which the left cell contains its index $i$ and the right cell contains its demand. There are two categories of link: strong links (bold arrows) are reliable and have a high bandwidth; weak links (dot-head arrows) are not reliable and have a low bandwidth.

To solve this problem, Mandriva's engineers combine solutions obtained by different heuristics to build a "balanced" solution. First, let's build two reliable solutions in which each pserver is connected to the root pserver located at the central facility by a reliable path. The first centralized solution consists in 5 pservers located at the central facility $(i=1)$. In this case, 400 clients benefit from local connections while the central facility provides 550 and 1550 connections to its left and right subtrees respectively. Figure 2(b) shows a solution as reliable but more efficient in which the facilities 1 and 3 respectively contain 2 and 3 pservers (indicated in the bottom cell). The link $(i, j)$ is now labeled with the number of connections provided by the pservers of facility $i$ to clients of the facility $j$. The dashed arrows are connections between a pair of facilities passing through a path rather than a single link. Thus, the total number of connections passing through link $(1,2)$ is the sum of connections of transfer paths $(1,2)$ and $(1,4)$. In this case, 1250 clients benefit from local connections and the central facility still provides 550 connections to its left subtrees. This solution provides more local connections and the broadcast is more efficient in the right subtree. The traffic passing through the link $(1,3)$ decreases from 1550 to 50 connections while the traffic passing through $(3,5)$ and $(3,6)$ remains unchanged. Moreover, several transfer paths have been shortened whereas no one has been lengthened.

On the contrary, the solution in Figure 2(c) favors 
efficiency rather than reliability. The facilities 1,2 , 5 contain a single pserver whereas the facility 3 contains 2 pservers. In this case, the pservers located at the facilities 2 and 5 are not connected to the central facility by a reliable path. However, 1850 clients benefit from local connections and only 650 connections pass through the network. The solution in Figure 2(d) reduces the number of connections passing through the network from 650 to 550 . Note that a further analysis is required to determine which of the two last solutions is preferred.

To conclude, it would be very difficult to express trade-offs between the reliability and the efficiency using a heuristic because these objectives are contradictory. Indeed, installing pservers in highest levels of the tree network gives solution more robust, scalable (if the numbers of clients increases), and reliable, but possibly less efficient.

\section{RELATED WORK}

Our problem is related to research about mixed integer linear programming, network design, cloud management, and facility location, especially server location in computer networks.

\section{Mixed Integer Linear Programming}

A mixed-integer program is the minimization or maximization of a linear function subject to linear constraints. Since more than 50 years, Mixed Integer Linear Programming (MILP) theory and practice has been significantly developed and is now an indispensable tool in Operations Research and Management Science (Guignard-Spielberg and Spielberg, 2005). Two reasons for the success of MILP are Linear Programming (LP) based solvers and the modelling flexibility of MILP. We now have several extremely effective state of the art solvers (IBM, 2012; Gurobi Optimization, 2012) that incorporate many advanced techniques and, since its early stages, MILP has been used to model a wide range of applications (Dantzig, 1960). Since then, MILP has been applied in different industrial application areas, such as supply chain management (Beamon, 1998), production planning (Wu and Shi, 2011), healthcare (Ferris et al., 2006), and airline fleet scheduling (Sherali et al., 2010).

\section{Network Design}

In the network design problem (Johnson et al., 1978), a weighted undirected graph is given. We wish to find a subgraph which connects all the original nodes and minimizes the sum of the shortest path weights between all nodes pairs, subject to a budget constraint on the sum of its edge weights. Usually, the cost of an edge is a concave function of its capacity. Additionally, there can be a demand between pairs of nodes of the network that corresponds to sending some product along a path between these two nodes. The quantities that pass through an edge are subject to an edge capacity constraint.

There is a relation between the bandwidth of PSLP and the edge capacity of the network design problem. However, it is also quite different because we need to determine the number of pservers, their types and locations. Moreover, the demand between pservers and clients has also to be determined whereas it is given in the network design problem. Last, the criteria combination used for PSLP is different because links already exist and it does not make any difference to use it or not.

\section{Cloud Management}

Cloud computing industry faces many decision problems where operations research could add tremendous value (Iyoob et al., 2012). Indeed, the paradigm shift from IT as a product to IT as a service and the accompanying flexibility gives rise to a vast array of resource management decisions. The flexibility and elasticity of cloud computing is enabled by virtualization, which allows to the control of computing resources to be physically separated from the resources themselves. There are three major players in the Cloud IT Supply Chain: a) providers (compute, storage, and network) b) consumers (individual and enterprises) c) brokers (equivalent of third-party logistics).

Cloud providers costs are primarily tied to their assets and the maintenance of these assets. Some of these strategic problems are related to PSLP as they involve capacity, location planning and service levels. But, they concern physical resources (servers, infrastructure, power draw, network) whereas we focus on the deployment of a distributed application.

The operational problem of assigning virtual machine to hosts subject to capacity constraints for several dimensions (CPUs, memories ...) is very different because the optimization criteria concern load balancing or scheduling. Consumer problems concern the capacity planning and scheduling of virtual machines in order to minimize subscription costs. Last, broker problems concern the provider's choice as well as inter-provider transformation and migration abilities. 


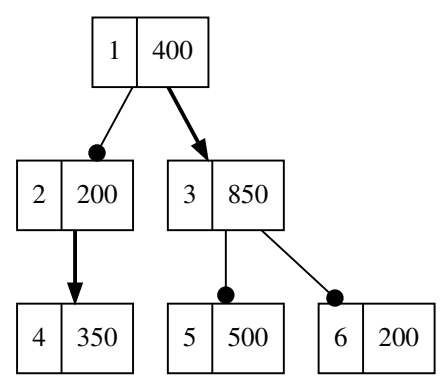

(a) Instance

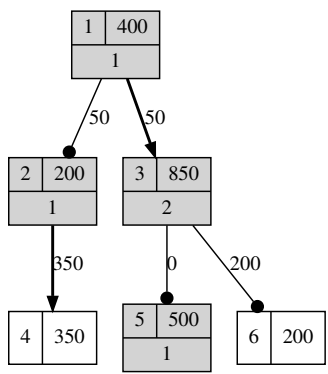

(c) Efficient solution

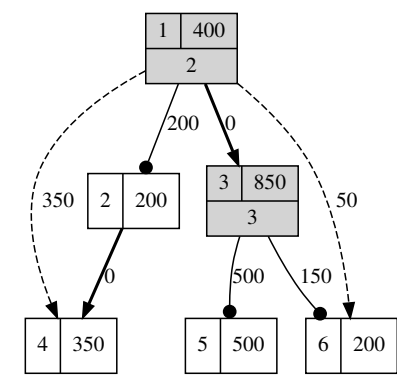

(b) Reliable solution

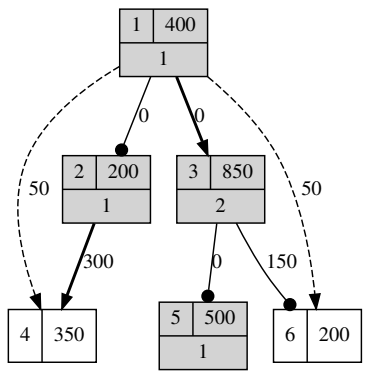

(d) More efficient solution?

Figure 2: Instance and solution examples.

\section{Facility Location}

In network location models (Berman and Krass, 2002), the underlying topology is that of a network, with the possible location of facilities including nodes, as well as edges of the network. These problems often extend the classical p-median and pcenter problems in different directions, including: incorporating interactions between facilities; considering multi-objective models, integrating location and routing decisions; and considering problems with robust decision criteria. In the p-center problem, we aim to minimize the maximum distance between a client and the facility to which he is allocated. In the pmedian problem, we aim to minimize the sum of the shortest demand weighted distance between clients and facilities. In both cases, each client selects the closest facility.

In discrete location models, the set of potential facility locations is discrete - often consisting of nodes of the underlying network. Due to the restricted location set, the underlying problem is somewhat easier - allowing for greater realism in the models. In stochastic demand/congestion models, this sub-class of discrete location models allows for demand to be stochastic and explicitly models congestion (queuing) at the facilities. In a stochastic environment, demand for service generated at nodes of the network are random variables and the time to service calls is also probabilistic. The location of servers has been ad- dressed as stochastic models (Berman and Drezner, 2007; Aboolian et al., 2009) in which servers are modeled as stochastic queues for which the theory enables the mathematical analysis of several related processes, including arriving at the queue, waiting in the queue, and being served at the front of the queue.

PSLP is strongly related to network location models because we want to choose locations hosting pserver in an existing network with opening costs. However, the optimality criteria are different, especially when it involves the concept of bandwidth. Furthermore, each client selects the closest facility in facility location models whereas a client is explicitly associated to a pserver by the central authority in the PSLP.

\section{MULTI-OBJECTIVE OPTIMIZATION}

Multi-objective optimization (or multi-objective programming or "pareto optimization") (Sawaragi et al., 1985; Steuer, 1986) also known as multi-criteria or multi-attribute optimization, is the process of simultaneously optimizing two or more conflicting objectives subject to certain constraints. Many interactive and non-interactive methods have been proposed for multi-objective mixed integer programming problems (Alves and Clímaco, 2007). In the last years, 
there have been many developments devoted to particular multi-objective combinatorial problems (such as location, scheduling, knapsack, shortest-path problems, etc). The researchers' attention has also focused on the use of meta-heuristics to solve these problems such as ant colony optimization, memetic algorithm, genetic algorithm, particle swarm optimization $(\mathrm{Hu}$ and Eberhart, 2002), and nested partitions (Wu et al., 2011).

\section{Multi-objective Methods without Trade-off}

The simplest approach, the lexicographic optimality, defines a first kind of trade-off: do not make any trade-off. It amounts to prefer any improvement of the most important criterion (objective), even a very small improvement, to any improvement of the second criterion, even a huge improvement. We have a strong hierarchical preference between criteria. In other words, the criteria are ordered lexicographically: a solution $s$ is better than $s^{\prime}$ if and only if it is equivalent for criteria $1, \ldots, i-1$, and is better for the $i$-th criterion. This approach has a great advantage, it does not need the values of the criteria to be compared. Indeed, the comparison of the values of different criteria is a difficult task that can be specific to a user and a problem. Another approach is simply to generate all the solutions which may be preferred. A solution may be preferred if and only if it is not dominated by another one. Such a non dominated solution is called a pareto-solution. A solution $s$ is better than $s^{\prime}$ if and only if for each criterion, $s$ is at least equivalent to $s^{\prime}$, and there exists a criterion such as $s$ is strictly better than $s^{\prime}$. Intuitively, we cannot improve a criterion of a pareto-solution without a loss for another criterion. In practice, the set of pareto solutions is huge, and the usual approach is to compute an approximation of this set.

\section{Multi-objective Methods with Trade-off}

The comparison of the values of different criteria is needed in order to define a good trade-off. In general, this comparison is made thanks to a utility function associated to each criterion.

A utility function $u_{z}$ maps a value $v$ of the criteria $z$ to a number $u_{z}(v)$. A utility function $u_{z}$ should satisfy an intuitive property: $v_{1}<v_{2} \Rightarrow u_{z}(v 1)<u_{z}(v 2)$. The aim of utility functions is to make possible the comparison between different criteria. The utility functions of different criteria are strongly related, in such a way that the numbers coming from different criteria can be compared: let $z_{1}, z_{2}$ be two criteria and $v_{1}$, $v_{2}$ be the values of $z_{1}, z_{2}$ in a given solution. We can know that, in this solution, the criterion $z_{1}$ is more favored than the criterion $z_{2}$ when $u_{z_{1}}\left(v_{1}\right)>u_{z_{2}}\left(v_{2}\right)$. Given the values $u_{1}, \ldots, u_{k}$ and $u_{1}^{\prime}, \ldots, u_{k}^{\prime}$ of the utility functions for two solutions $s$ and $s^{\prime}$, we want now to define when $s$ is preferred to $s^{\prime}$, noted $s>_{m} s^{\prime}$. Many different preference orders $>_{m}$, and many corresponding multi-criteria methods exist. We will consider the following methods.

Weighted Sum. An extremely frequent approach is to maximize a weighted sum $\sum w_{i} \times u_{i}$ where $u_{1}, \ldots, u_{k}$ are weights associated to each criterion. This approach seems to be quite intuitive and satisfactory. Nevertheless, despite its general use in many domains, this approach has some strong drawbacks. The function $\sum w_{i} \times u_{i}$ is linear, and, when maximizing this function for a concave set of solutions, many good solutions are not reachable. Furthermore, its sensitivity to the weights is very high.

Maximizing the Minimum. Another frequent approach is to maximize the minimum value among the criteria. $s>_{m} s^{\prime}$ iff $\min u_{i}>\min u_{i}^{\prime}$. This approach unfortunately produces many indistinguishable solutions, and some of them are not pareto solutions.

\section{Multi-objective Methods with Trade-off and Dynamic Ordering of the Criteria}

The weighted sum method, as well as the method of maximizing the minimum have clear drawbacks. Indeed, these two methods can be greatly improved by integrating in them the following idea: order the criteria depending on the values they take in a solution. Then, aggregation of the criteria can be done depending on the rank of the criteria. Let $p_{1}, \ldots, p_{k}$ be the sorted permutation of $u_{1}, \ldots, u_{k}$ in increasing order of values.

Ordered Weighted Average. Let $w$ be a vector of weights such that $w_{i} \geq w_{i+1}$. The idea is to maximize $\sum w_{i} \times p_{i}$. We can see that if $w_{1}=w_{2}=\ldots=w_{k}$, we get the sum. If $w_{1}=1, w_{2}=w_{3}=\ldots=0$, we get the methods which maximize the minimum. This method has been introduced by (Yager, 1988).

Leximin/Leximax. A leximin solution is simply a lexicographic optimal solution on $p_{1}, \ldots, p_{k}$. Leximin/leximax have nice properties, that make them intuitive for the user: a) they produce only pareto solutions; b) they are egalitarist (leximin solutions are always solutions for the method which maximizes the minimum); c) they verify the property of reducibility (if a criteria is set to a value it has in an optimal solution, we get the same set of optimal solutions). This 
method has been introduced in social choice community and then used for multi-objective optimization (Ehrgott, 2000).

\section{MATHEMATICAL FORMULATION}

Let's now define a MILP formulation of the problem. Let $P\left(i_{1}, i_{n}\right)=\left[i_{1}, \ldots, i_{p}, \ldots, i_{n}\right]$ denote the unique path of the tree network $\mathcal{T}$ between $i_{1}$ and $i_{n}$ if any.

Location of the Pservers. Let $x_{i k}$ denote the number of pservers of type $k \in K$ located at the facility $i \in N$. Constraints (1) impose a maximal number $M_{i k}$ of pservers of type $k$ located at facility $i$.

$$
x_{i k} \leq M_{i k} \quad \forall i \in N, \forall k \in K
$$

Traffic Flow. Let $y_{i}^{s}$ denote the number of connections provided to clients by pservers located at the facility $i \in N$ for the stage $s \in S$. Let $y_{i j}^{s}$ denote the number of connections passing through the link $(i, j) \in E$ for the stage $s$. Constraints (2) enforce that the number of connections provided by the facility $i$ stays below the cumulated capacity of its pservers. Constraints (3) enforce the flow conservation of connections at facility $j$, i.e. incoming $\left(y_{i j}^{s}\right)$ and provided $\left(y_{j}^{s}\right)$ connections satisfy the demand $\left(d_{j}^{s}\right)$ while enough outgoing connections $\left(y_{j p}^{s}\right)$ remain to serve its descendants.

$$
\begin{array}{lr}
\sum_{k \in K} C_{k} x_{i k} \geq y_{i}^{s} & \forall i \in N, \forall s \in S \\
\sum_{(j, p) \in E} y_{j p}^{s}=y_{i j}^{s}+y_{j}^{s}-d_{j}^{s} & \forall(i, j) \in E, \forall s \in S
\end{array}
$$

These constraints are illustrated in Figure 3 for a facility $j$ with three children $p_{1}, p_{2}$ and $p_{3}$ (the stage is omitted).

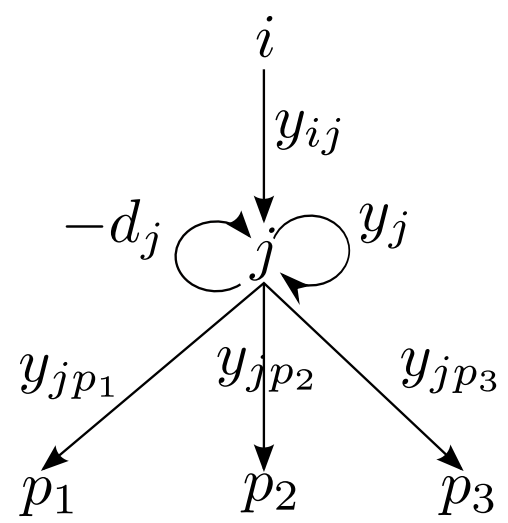

Figure 3: Traffic flow.
Client-server Model. Let $z_{i}^{s}$ denote the number of local connections at the facility $i$ for the stage $s$. Constraints (4) enforce that the number of local connections at the facility $i$ is equal to the demand minus the number of incoming connections (from its ancestors). Let $z_{i j}^{s}$ denote the number of connections from facilities $i$ to $j$ for the stage $s$, i.e. passing through the transfer path $P(i, j)$. Constraints (5) enforce that the number of connections passing through the link $(i, j)$ is equal to the total number of connections of transfer paths taking this link.

$$
\begin{aligned}
z_{i}^{s} & =d_{i}^{s}-\sum_{p \in A(i)} z_{p i}^{s} \quad \forall i \in N, \forall s \in S \\
y_{i j}^{s} & =\sum_{(i, j) \in P(u, v)} z_{u v}^{s} \quad \forall(i, j) \in E, \forall s \in S
\end{aligned}
$$

Broadcast Model. Let $b_{i j}^{s}$ denote the path bandwidth from facilities $i$ to $j$ for the stage $s$. Constraints (6) enforce that the sum of path bandwidths consumed at the link $(i, j)$ is lower than its capacity. Let $B_{\min }$ and $B_{\max }$ be respectively the minimum and maximum bandwidth allocated to a single connection from a pserver to a client. The central authority determines their values depending on the network architecture (clients, pservers and links). Constraints (7) and (8) enforce that the bandwidth per connection between facilities $i$ and $j$ belongs to a given range.

$$
\begin{array}{ll}
\sum_{(i, j) \in P(u, v)} b_{u v}^{s} \leq B_{i j} & \forall(i, j) \in E, \forall s \in S \\
b_{i j}^{s} \geq B_{\min } \times z_{i j}^{s} & \forall(i, j) \in E, \forall s \in S \\
b_{i j}^{s} \leq B_{\max } \times z_{i j}^{s} & \forall(i, j) \in E, \forall s \in S
\end{array}
$$

Initial Broadcast. The last constraints handle the broadcast from the root pserver to other pservers, i.e. the stage $s=0$. Constraint (9) enforces that the root pserver, which broadcasts packages to other pservers, is located at the central facility $(i=1)$. Constraints (10) enforce that demand at a facility $i$ for the stage 0 is equal to its number of pservers. Constraints (11) enforce that the root pserver serves all other pservers.

$$
\begin{array}{lr}
x_{11} \geq 1 & \\
d_{i}^{0}=\sum_{k \in K} x_{i k} & \forall i \in N \\
y_{i}^{0}=z_{i j}^{0}=b_{i j}^{0}=0 \quad \forall i \in N \backslash\{1\}, \forall j \in N
\end{array}
$$

\section{COMBINING MULTIPLE OBJECTIVES}

The criteria combination methods introduced in Sec- 
tion 4 offers much more possible criteria combinations than expected. We refer the reader to (Guttierez et al., 2011) for an explanation of how to handle multiple criteria in an integer programming framework. Their approach is the basis of a flexible system of criteria combination which provides a useful mean to address some user's needs. A key observation is that all these combinations can be viewed as a variation of the lexicographic algorithm.

We describe here a general approach for lexicographic optimization that can be implemented on top of any existing optimization solver. The underlying optimization solver is only required to be able to maximize an objective function $F$ subject to the set of constraints $C$. Let $\max (F, C)$ be the minimal value of $F$ among all the feasible solutions of the constraint system $C$. This optimal value is computed by calling the underlying optimization solver. The lexicographic optimization problem amounts to maximize lexicographically $n$ criteria $F_{1} \ldots, F_{n}$ while satisfying a set of constraints $C$. A lexicographic optimization problem $P$ can be solved by solving a sequence of (single criterion) optimization problems $\left\{P_{i}\right\}_{i \in[1, n]}$ with an underlying optimization solver. Each problem $P_{i}$ consists of maximizing $F_{i}$ while satisfying the constraints $C_{i}$ where: a) $C_{1}=C$, b) $\forall i \in[2, n], C_{i}=$ $C_{i-1} \cup\left\{F_{i-1}=\max \left(F_{i-1}, C_{i-1}\right)\right\}$ The solution of the optimization problem $P_{n}$ is the solution of the lexicographic optimization problem $P$. The leximin/leximax optimization relies on a similar approach.

A Language for User Defined Combination. We provide a small dedicated language to let the user define its own criteria combination. The key idea is to map, at least, virtually, any possible criteria combination to an underlying lexicographic order. Indeed, mono-objective criteria combinations, like a simple aggregation, fits directly to a lexicographic algorithm reduced to one criteria and the leximin/leximax order implementation do rely on an underlying lexicographic order implementation. Therefore, the basic criteria combination is the lexicographic one:

-lexicographic $[\langle$ lccriteria $\langle\{,\langle$ lccriteria $\rangle$ * $]$

-lex $[<$ lccriteria $>\{,<$ lccriteria $\rangle$ * ]

The next level of criteria combination gives access to the leximin/leximax order:

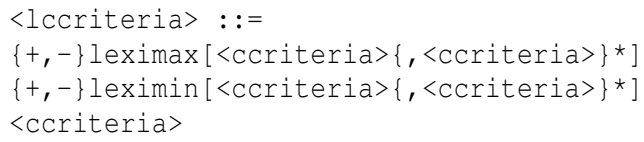

As a consequence, one, two or more leximin/leximax orders can be lexicographically ordered, meaning that, for instance, the user does not want to or- der the two first criteria, neither he wants to order the third and fourth criteria, while he clearly wants the set of the two first criteria to be handled before the rest of the criteria. Note that the leading sign gives the optimization direction, i.e., a leading - for a minimization and a leading + for a maximization.

The final level of combiners gives access to the agregate and lexagregate combination:

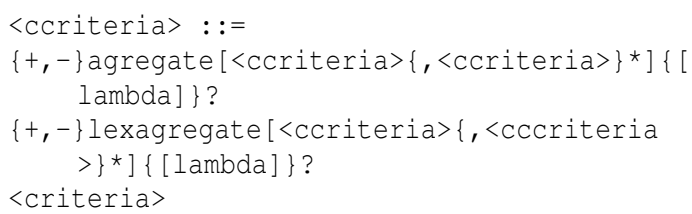

This recursive definition allows the user to define agregate of agregate or lexagregate. The lexagregate combiner uses only one objective function which could be solved using the lexicographic algorithm. An optional lambda value gives the opportunity to attach a weight to the agregate by means of a positive integer. All coefficients of the criteria will be multiplied by this weight. At last, the user can choose among the predefined criteria which one he wants to optimize:

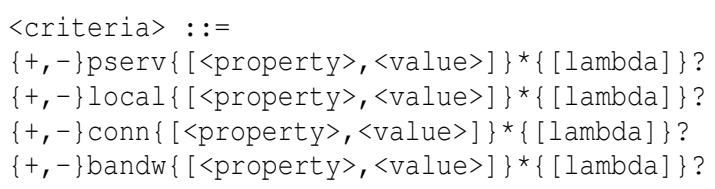

The language allows to specify a range property as a single integer value or an interval matching the following regular expression: $\langle$ range $>$ : : =int $(-\mid-i n t)$ ?. So, the user can specify a subset of facilities $N^{\mathrm{c}}$ by using [ level, <range> ] which restricts to facilities located at given levels of the network and [reliable, <bool $>$ ] which restricts to facilities for which the path from the central facility are (non-)reliable. Similarly, the user can specify a subset of pserver types or stages by using [type, <range>] and [stage, <range>] respectively. Last, the user can restrict the length of transfer paths (number of arcs) by using [ length, <range> ] and select only (non-)reliable paths by using [reliable, $<$ bool $>$ ]. If a property is not given, then the condition is satisfied.

\section{Predefined Criteria}

Several criteria are predefined. Let $N^{\mathrm{c}} \subseteq N, K^{\mathrm{c}} \subseteq K$, $S^{\mathrm{c}} \subseteq S$ and $P^{\mathrm{c}} \subseteq N^{2}$ respectively be some subsets of the facilities, pserver types, stages and paths specified by the user.

The pserv criterion counts the number of pservers for a subset of facilities and for a subset of pserver 
types:

$$
\sum_{i \in N^{\mathrm{c}}} \sum_{k \in K^{\mathrm{c}}} x_{i k}
$$

The local criterion counts the number of local connections at a subset of the facilities for a subset of stages:

$$
\sum_{i \in N^{\mathrm{c}}} \sum_{s \in S^{\mathrm{c}}} z_{i}^{s}
$$

The conn criterion counts the number of connections for a subset of the transfer paths and for a subset of stages:

$$
\sum_{(i, j) \in \operatorname{Pc}^{\mathrm{c}}} \sum_{s \in S^{\mathrm{c}}} z_{i j}^{s}
$$

The bandw criterion sums the path bandwidths for a subset of the transfer paths and for a subset of stages:

$$
\sum_{(i, j) \in \mathrm{P}^{\mathrm{c}}} \sum_{s \in S^{\mathrm{c}}} z_{i j}^{s}
$$

Examples. The listing 1 gives aliases for criteria combinations used in Section 7. First, we define a linear cost function cost_pserv associated to the number of deployed pservers of each type by using the agregate combiner. Another agregate combiner dist_pserv allows to sum the lengths of transfer paths as in facility location models. The sum_bandw criterion estimates the total bandwidths allocated to the broadcast by distinguishing local and remote connections. The capa_pserv criterion computes the total capacity of the deployment whereas the bad_pserv criterion returns a vector with the number of non-reliable, new and "proxy" pservers.

\section{EXPERIMENTAL RESULTS}

This section presents computational experiments conducted to evaluate our approach. First, we describe a method for generating realistic instances on which the experiments have been done. Then, a use case shows that playing with criteria combination allows to exhibit different solutions which are then improved by incrementally refining the criteria combination. Last, the performance of our approach is evaluated depending on the size of the instances as well as the criteria combination.

An application provides a MILP platform to solve PSL problems. It translates a PSL problem and a set of criteria in one or more integer programs that are then solved by an underlying integer programming solver. It has been developed at UNS in C++ and is available at http://github.com/arnaud-m/opossum under a modified BSD license. It actually offers interfaces to two integer programming solvers: IBM
Ilog CPLEX (IBM, 2012), GLPK (GLPK, 2012). All the experiments were conducted on a Linux machine, with $32 \mathrm{~GB}$ of RAM and 6 processors $(3.2 \mathrm{GHz})$ with CPLEX 12.3.0 (mono-thread).

\section{Generating Realistic Instances}

Mandriva's team have provided useful information to generate realistic instances. First, the tree networks have about four levels. In complex scenario, the average numbers of facilities and clients are equal to 1000 and 100000 respectively. The characteristics of a facility are defined by its type (about ten types) which is associated to a level of the network. We consider two groups of clients, i.e. the problem has three stages. We assume that the bandwidth of links is one of the following: 2MB (low-end DSL), 20MB (high-end DSL), 100MB (low-end LAN), 1GB (lowend LAN).

There are five types of servers which provide different number of connections. The best alternative is to install package servers on existing Unix servers of the network, but we can buy and install new Unix servers $\left(C_{1}=C_{2}=500\right)$. The pservers can also be installed on Unix $\left(C_{3}=300\right)$ and Windows $\left(C_{4}=100\right)$ clients, but they provide less connections and are more expensive. As a last resort, it is possible to activate the "proxy mode" of any client $\left(C_{5}=20\right)$ which is thereby temporary transformed, at great expense, into a pserver with limited capacity.

There are twelve types of facility characterized by their demands and server capacities as well as some probabilities used for the network generation. The generation of the tree network relies on a breadth-first algorithm. For a given node, its children are generated as follows. First, the number of facilities is drawn randomly for each type of the next level using a binomial distribution $B(n, p)$. Second, the bandwidth and reliability of the links between the node and its children are drawn randomly using uniform distributions. Additionally, we can enforce that the structure of the network is strictly hierarchical: a link has a lower bandwidth and is less reliable than its predecessors. In this case, the bandwidth probabilities are normalized according to the bandwidth of its predecessor in order to fulfill the hierarchical condition.

\section{Use Case}

We discuss a realistic use case based on a synthetic instance for which we apply two scenarios. The network is composed of 769 facilities and 24320 clients. There is a total of eight available Unix servers and it is possible to buy at most one new Unix servers at each facility of levels 0 and 1 (a total of 16 new 


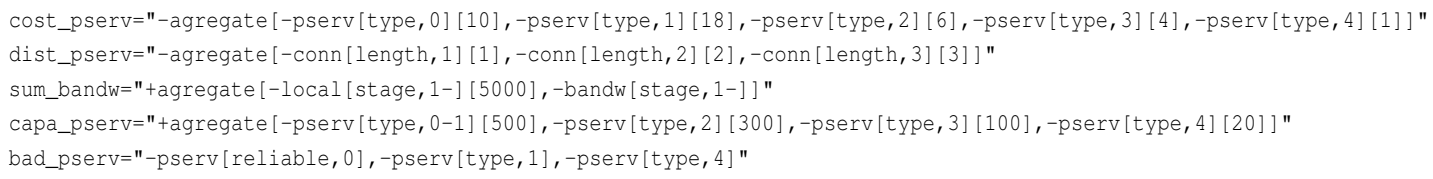

Listing 1: Aliases of several criteria combinations.

Unix servers). In the first scenario, we aim to minimize the number of deployments while maximizing the reliability but also taking into account preferences between pserver types. In this scenario, the broadcast model only checks the feasibility constraints, i.e. the minimal bandwidth allocated to a single client. In the second scenario, we aim to minimize a cost function associated to the deployment while maximizing the broadcast performance.

The user must deploy at least 30 pservers spread over 16 facilities (-lex [-pserv] ). The solution vector $(8,14,8,0,0)$ is composed of 8 Unix servers, 14 new Unix servers, 8 Unix clients, 0 Windows clients, and 0 proxy clients. The spare capacity of a deployment is defined as the minimal spare capacity among groups. So, the user determines the maximal spare capacity equal to $6.2 \%$ (-lex [-pserv, capa_pserv]) whereas it was only equal to $3.2 \%$ in the previous solution. Now, the user want to increase the reliability of the deployment modeled by the bad_pserv criteria. The user first determines an egalitarist solution (-lex[-pserv,-leximax[bad_pserv]]) in which the number of reliable pservers is improved from 22 to 24 and the number of new Unix servers is reduced from 14 to 12 . The solution vector is now $(8,12,10,0,0)$, but the deployment has a low spare capacity of $0.5 \%$. Then, the user determines an alternative solution $(-1$ ex $[-$ pserv, bad_pserv]) in which it is preferred to increase the number of reliable pservers (from 22 to 25 ) rather than reducing the number of new Unix servers (from 14 to 13). This last solution $(8,13,9,0,0)$ is preferred to the previous one because of its better spare capacity (2\%). In all previous solutions, pservers are spread over facilities of the levels 1 and 2 .

The user must deploy 41 pservers spread over 20 facilities for a total cost of 284 (lex [ cost_pserv]). The solution $(7,2,28,2,2)$ uses all types of pservers and has a low spare capacity $(1.5 \%)$. From now on, pservers are spread over facilities of the levels 1 to 3. Note that the two best solutions of the first scenario have a cost of 356 and 368 respectively. Then, the user determines that improving the reliability (lex[cost_pserv, bad_pserv] or lex[cost_pserv,-leximax[bad_pserv] ]) only results in increasing the number of reliable pservers from 27 to 31 while leaving the solution vector unchanged. Finally, the user prefers to improve the broadcast performance (lex[cost_pserv, sum_bandw] or lex [cost_pserv, dist_pserv]) which changes the solution vector to $(6,2,29,3,2)$. The 42 pservers are spread over more facilities (21) and more levels (1-3) than in the first scenario, but has less spare capacity $(0.8 \%)$

Note that solving times significantly depend on criteria combinations. Indeed, solving times of the first scenario remain lower than 5 seconds whereas those of the second scenario increases until 5 minutes (from the second to the fourth criteria combinations). Intuitively, subproblems with a minimal number of pservers are easier to solve than those with a minimal cost because there are less feasible deployments.

This use case has showed that our approach can provide a flexible and efficient decision support tool for the deployment of package servers in a tree network. In the next section, we will evaluate the performance and benefits of our approach in a systematic way.

\section{Performance Evaluation}

Our approach has been tested on 40 synthetic instances. The number of facilities ranges from 300 to 1700 with the average around 1000 . The total demand ( 2 groups) ranges from 10000 to 50000 . The average number of existing and new Unix servers is 33 .

We discuss here the impact of the criteria combination on the solving time of the problem. We compare families of combinations which concern similar aspects of the deployment. Then, we study the influence of the criteria ordering before a comparison of the primary criterion (see below). Let recall that the listing 1 page 10 gives aliases for several criteria combinations. The basic criteria combination is-lex [ (criteria, lccriteria]. The primary criteria is either-pserv or cost_pserv in order to restrict the number of pservers. The Secondary lccriteria are presented gradually and concern the efficiency and the reliability. For each of 24 criteria combinations, instances were tested with a time limit set to 1000 seconds per subproblems. The number of subproblems depends only of the criteria combination. 835 of 960 problems were optimally solved. The graphics of Figure 4 show percentages of instances 


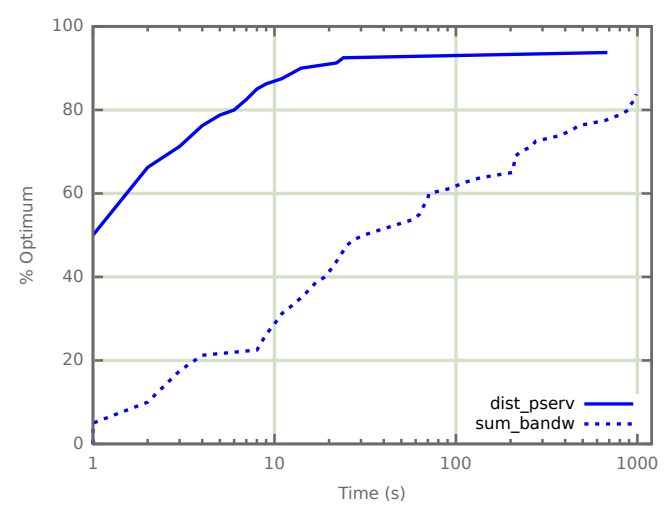

(a) Distance versus bandwidth

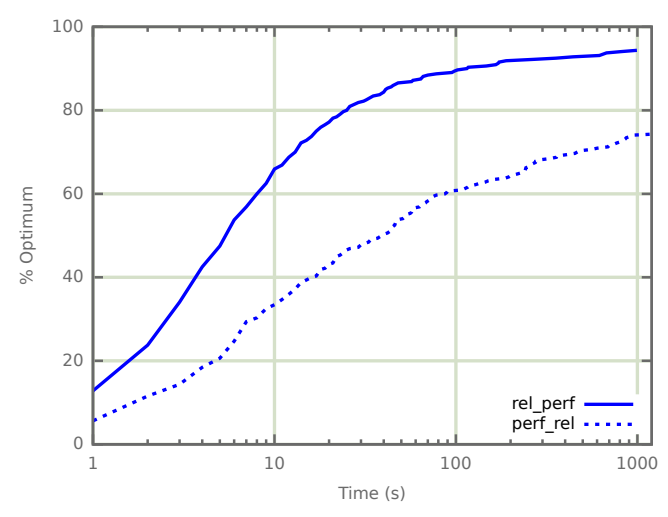

(c) Reliability versus performance

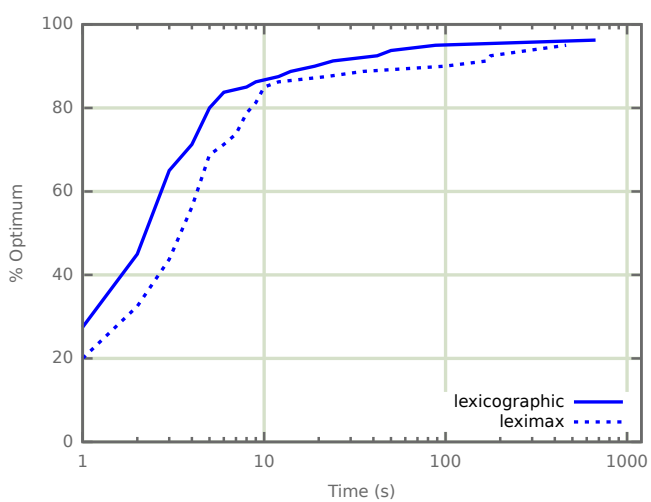

(b) Lexicographic versus leximax

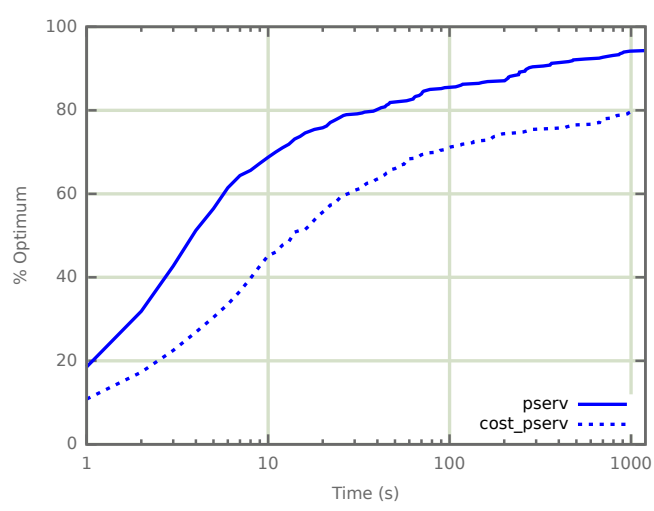

(d) Size versus cost

Figure 4: Experimental results for criteria combinations.

solved optimally as a function of the time in seconds for groups of criteria combinations.

Distance versus Bandwidth. We compare bicriteria combinations related to the efficiency of the broadcast. Figure 4(a) compares the resolution of problems where lccriteria is either sum_bandw or dist_pserv with both primary criteria. More than $80 \%$ of the dist_pserv problems are solved within 10 seconds whereas only about $30 \%$ of sum_bandw problems. The solver takes advantage of additional time for solving up to $80 \%$ of the sum_bandw problems within 1000 seconds whereas the resolution of the dist_pserv problems has reached a floor around 20 seconds. So, the dist_pserv criterion can be used for discovering quickly good solution before using the sum_bandw criterion for solution refinement.

Lexicographic versus Leximax. We compare 4criteria combinations related to the reliability of the broadcast. Figure 4(b) compares the resolution of problems where lccriteria is either bad_pserv or - leximax [bad_pserv] with both primary criteria. It is worthwhile to use leximax to smooth the values of the criteria. More than $80 \%$ of the problems are solved within 10 seconds for both criteria and the results are similar.

Reliability versus Performance. Now, we evaluate the impact on the solving time of the relative ordering between the efficiency and reliability criteria. Figure 4(c) compares the resolution of problems where, for instance, lccriteria is on the one hand bad_pserv, sum_bandw (rel_perf) and on the other hand sum_bandw, bad_pserv (perf_rel). The results indicate that is preferable to improve the reliability of the deployment, and then its efficiency.

Size versus Cost. Last, Figure 4(d) compares the resolution of problems depending on the primary criterion for all secondary criteria described above. The results confirm the intuition that pserv subproblems where the number of pservers is fixed are more constrained (and easier) than cost_pserv subproblems where only the installation cost is fixed. Though, cost_pserv problems allow to discover more various solutions by playing with criteria combinations. 


\section{CONCLUSIONS}

We have introduced a new multi-objective optimization problem derived from a real-world application: the package server location problem. We have proposed a first mathematical formulation of this multiobjective optimization problem as well as a method for the generation of realistic instances. In addition, a small dedicated language allows virtually to define any possible criteria combination.

Results for exact multi-objective solution approaches based on mixed integer linear programming have been reported. First, a realistic use case has shown the efficiency and flexibility of our approach for quick prototyping of deployments with various structural properties. Second, solving times are really satisfactory for realistic problem's sizes. Last, some criteria combinations are "easier" to solve than others to obtain solutions with similar characteristics.

In further work, we will compare different linear programming solvers, and investigate approximations of the pareto set.

\section{ACKNOWLEDGEMENTS}

The authors would like to thank Claude Michel, Mohamed Rezgui (UNS CNRS), and Yvan Manon (Mandriva). This work was supported by the Agence Nationale de la Recherche (Aeolus project - ANR-2010SEGI-013-01). To end, we would like to thank the anonymous referees for their remarks.

\section{REFERENCES}

Aboolian, R., Berman, O., and Drezner, Z. (2009). The multiple server center location problem. Annals of Operations Reseach, 167:337-352.

Alves, M. J. and Clímaco, J. (2007). A review of interactive methods for multiobjective integer and mixed-integer programming. European Journal of Operational Research, 180(1):99 - 115.

Beamon, B. M. (1998). Supply chain design and analysis:: Models and methods. International Journal of Production Economics, 55(3):281 - 294.

Berman, O. and Drezner, Z. (2007). The multiple server location problem. The Journal of the Operational Research Society, 58(1):91-99.

Berman, O. and Krass, D. (2002). Recent Developments in the Theory and Applications of Location Models: A Preview. Annals of Operations Reseach, 111:15-16.

Dantzig, G. B. (1960). On the significance of solving linear programming problems with some integer variables. Econometrica, 28:30-44.
Ehrgott, M. (2000). Multicriteria optimization. SpringerVerlag.

Ferris, M., Meyer, R., and D'Souza, W. (2006). Radiation Treatment Planning: Mixed Integer Programming Formulations and Approaches. In Appa, G., Pitsoulis, L., and Williams, H., editors, Handbook on Modelling for Discrete Optimization, volume 88 of International Series in Operations Research \& Management Science, pages 317-340. Springer US.

GLPK (2012). GNU Linear Programming Kit. http:// www. gnu.org/software/glpk.

Guignard-Spielberg, M. and Spielberg, K., editors (2005). Integer Programming: State of the Art and Recent Advances-Part I, volume 139. Annals of Operations Reseach.

Gurobi Optimization (2012). The Gurobi Optimizer. http: //www. gurobi.com.

Guttierez, G., Janota, M., Lynce, I., Lhomme, O., Manquinho, V., Marques-Silva, J., and Michel, C. (2011). Final version of the optimizations algorithms and tools. Technical report, Project Mancoosi: Managing the Complexity of the Open Source Infrastructure.

Hu, X. and Eberhart, R. (2002). Multiobjective Optimization Using Dynamic Neighborhood Particle Swarm Optimization. In Congress on Evolutionary Computation (CEC'2002), volume 2, pages 1677-1681, Piscataway, New Jersey. IEEE Service Center.

IBM (2012). IBM Ilog Cplex Optimizer. http: //www-01.ibm.com/software/integration/ optimization/cplex-optimizer/.

Iyoob, I., Zarifoglu, E., and Dieker, A. B. (2012). Cloud computing operations research. Service Science, Accepted.

Johnson, D. S., Lenstra, J. K., and Kan, A. H. G. R. (1978). The Complexity of the Network Design Problem. Networks, 8:279-285.

Sawaragi, Y., Nakayama, H., and Tanino, T. (1985). Theory of Multiobjective Optimization. Mathematics in Science and Engineering. Academic Press.

Sherali, H. D., Bae, K.-H., and Haouari, M. (2010). Integrated Airline Schedule Design and Fleet Assignment: Polyhedral Analysis and Benders' Decomposition Approach. INFORMS Journal on Computing, 22(4):500.

Steuer, R. E. (1986). Multiple Criteria Optimization: Theory, Computation and Application. John Wiley, New York.

Wu, T. and Shi, L. (2011). Mathematical models for capacitated multi-level production planning problems with linked lot sizes. International Journal of Production Research, 49(20):6227-6247.

Wu, T., Shi, L., Geunes, J., and Akartunali, K. (2011). An optimization framework for solving capacitated multilevel lot-sizing problems with backlogging. European Journal of Operational Research, 214(2):428 - 441.

Yager, R. R. (1988). On ordered weighted averaging aggregation operators in multicriteria decision making. Systems, Man and Cybernetics, IEEE Transactions on, 18:183-190. 